\title{
Considerações sobre o ensaio de retorno elástico por torção para asfaltos modificados
}

\author{
Considerations about the torsion recovery test of modified binders
}

\author{
Thiago Hilário da Cruz ${ }^{1}$, Thiago Seliger Woellner ${ }^{2}$, Lilian Tais de Gouveia ${ }^{3}$, Luciano José Senger ${ }^{4}$ \\ 1Universidade Estadual de Ponta Grossa, Paraná - Brasil, thiagohlro@outlook.com \\ 2Universidade Estadual de Ponta Grossa, Paraná - Brasil, thiagowoellner@icloud.com \\ 3Universidade Estadual de Ponta Grossa, Paraná - Brasil, Itgouveia@uepg.br \\ 4Universidade Estadual de Ponta Grossa, Paraná - Brasil, ljsenger@uepg.br
}

\section{Recebido:}

8 de março de 2018

Aceito para publicação:

10 de setembro de 2019

Publicado:

31 de agosto de 2020

Editor de área:

Francisco Thiago Aragão

\section{Palavras-chaves:}

Retorno elástico por torção.

Asfaltos modificados.

Repetibilidade e reprodutibilidade.

\section{Keywords:}

Elastic recovery test.

Modified binders.

Repeatability and reproducibility.

DOI:10.14295/transportes.v28i3.1974

\section{RESUMO}

O objetivo deste trabalho é analisar o ensaio de Retorno Elástico por Torção (NLT329/91) quanto ao tempo de giro da amostra de asfalto e quanto ao controle da temperatura de banho do asfalto durante o retorno elástico, assim como a Repetibilidade e a Reprodutibilidade. $O$ ensaio de Retorno Elástico por Torção avalia a recuperação elástica dos asfaltos e consiste em submeter uma amostra de asfalto à uma torção de $180^{\circ}$, sendo o giro de torção realizado em um intervalo de $3 \mathrm{~s} \mathrm{a} 5 \mathrm{~s}$. O resultado do ensaio é expresso em porcentagem de recuperação elástica, após 30 minutos de retorno elástico. Neste estudo, foram utilizados três tipos de ligantes modificados. Os ensaios foram executados por dois operadores em tempos de giro entre $3 \mathrm{~s}$ e $5 \mathrm{~s}$ e entre $30 \mathrm{~s}$ e $50 \mathrm{~s}$, com e sem controle de temperatura de banho da amostra durante o tempo de retorno elástico. Os resultados mostraram que o tempo de giro influencia os resultados de retorno elástico. Quanto maior o tempo de giro, menor o retorno elástico. A pequena variação da temperatura de banho do asfalto durante o tempo de retorno elástico não influenciou os resultados. No entanto, recomenda-se que a temperatura de banho seja mantida constante. A Repetibilidade, mesmo operador e mesmo equipamento, e Reprodutibilidade do ensaio se mostraram elevadas, ou seja, as diferenças entre resultados replicados por um mesmo operador, assim como as diferenças de resultados replicados entre operadores distintos, apresentaram variabilidade baixa. A variação total foi de $3,5 \% \mathrm{em}$ relação à média e desvio padrão médio de 2,3.

\section{ABSTRACT}

The objective of this work is to analyze Elastic Recovery Test (NLT-329/91) regarding the time of rotation of the asphalt sample and the control of the bath temperature of the asphalt during the elastic return, as well as the Repeatability and Reproducibility. Elastic Recovery Test evaluates the elastic recovery of asphalts and consists of subjecting an asphalt sample to a torsion of $180^{\circ}$ in a range of $3 \mathrm{~s}$ to $5 \mathrm{~s}$. The test result is expressed as percentage of elastic recovery after 30 minutes. Three types of modified binders were used. The tests were performed by two operators in spin times between $3 \mathrm{~s}$ and $5 \mathrm{~s}$ and between $30 \mathrm{~s}$ and $50 \mathrm{~s}$, with and without water bath temperature control during the elastic return time. The results showed that torsion time influences the elastic return results. The longer the torsion time, the lower the elastic return. Variation of the water bath temperature of the asphalt during the elastic return time did not influence the results. Repeatability and Reproducibility of the assay were high. The differences among results replicated by the same operator and among different operators were of low variability. The total variation was $3.5 \%$ in relation to the mean and standard deviation of 2.3 . 


\section{INTRODUÇÃO}

0 modal rodoviário é responsável por mais de $60 \%$ do transporte de cargas e de $90 \%$ do transporte de passageiros no país. Apenas $12,3 \%$ das rodovias brasileiras são pavimentadas, destas, 32\% apresentam superfície do pavimento classificada como "Perfeita", já 49\% encontram-se desgastadas, $15 \%$ apresentam trincas em malha e/ou remendos mal executados, em $3 \%$ foram observados afundamentos, ondulações e buracos em conjunto ou isoladamente, e 1\% estão destruídas. Entre outros problemas, a condição dos pavimentos aumenta o custo operacional do transporte de cargas. Estima-se que só em 2016, em razão da má condição do pavimento, o transportador gastou $\mathrm{R} \$ 2,34$ bilhões a mais, custo esse que é repassado no custo das mercadorias e promove desvantagem competitiva no mercado internacional. Além do fator econômico, uma boa qualidade da malha rodoviária gera conforto e segurança aos usuários (CNT, 2017).

0 referido estudo da CNT, sobre a baixa durabilidade de pavimentos asfálticos no Brasil, aponta entre outros fatores, as deficiências no controle de qualidade de matérias-primas como um dos motivos para o aparecimento de defeitos em pavimentos, especialmente nos novos. Os defeitos mais comuns encontrados são o trincamento por fadiga e a deformação permanente (BERNUCCI et al.,2008).

A utilização de ligantes asfálticos modificados pode elevar a vida de serviço do pavimento e reduzir a frequência das manutenções, o que é importante principalmente em vias de difícil acesso ou onde o custo de paralisação do tráfego para que sejam feitas correções é muito elevado, por exemplo em pontes, viadutos e corredores de ônibus. Existem diversos tipos de modificadores de asfalto no mercado nacional atualmente, incluindo o copolímero em bloco Estireno Butadieno Estireno (SBS), o terpolímero reativo (Reactive Elastomeric Terpolymer - RET) e a borracha de pneu moído. Os modificadores de asfalto melhoram certas propriedades das misturas, incluindo ponto de amolecimento, viscosidade, força de coesão, ductilidade e especialmente a recuperação elástica do ligante asfáltico (BATES \& WORCH,1987; SILVA et al., 2002).

A recuperação elástica, também chamada de retorno elástico, é um parâmetro indicador do comportamento do material à deformação, pois avalia o grau em que uma substância recupera sua forma original após cessada a solicitação de carga (YILDIRIM, 2007). No Brasil, a recuperação elástica é avaliada utilizando o método de Recuperação Elástica pelo Ductilômetro, regulamentado pela norma 130/2010 - ME do DNIT e NBR 15086. O ensaio é composto por vários procedimentos, sendo necessárias mais de $4 \mathrm{~h}$ para sua realização, entre a preparação da amostra e obtenção do resultado. 0 retorno elástico (RE) é obtido pelo valor médio de três determinações de retorno elástico para uma mesma amostra, em \%.

Em alguns países, a recuperação elástica é analisada por torção utilizado o torciômetro (Figura 1). 0 ensaio de Retorno Elástico por Torção foi desenvolvido na Espanha pelo Centro de Estudios de Carreteras (Madri) e pelo Centro de Estudios y Experimentación de Obras Públicas, regulamentado pela norma NLT 329/91. Trata-se de um método mais simples e rápido para obtenção do retorno elástico, além de exigir um equipamento mais barato se comparado ao ductilômetro. A norma NLT 329/91 não tem correspondente brasileira, embora distribuidoras nacionais de asfalto realizem esse ensaio para asfaltos modificados destinados à exportação. Não há na norma espanhola, nem foi encontrado na escassa literatura sobre o assunto, dados sobre a precisão do ensaio, nem sobre sua repetibilidade e reprodutibilidade. 
Em um passo a passo, o ensaio consiste em:

- Montar o cilindro de torção, deixando a sua base a $20 \mathrm{~mm}$ do fundo da cápsula.

- Aquecer a amostra de asfalto modificado até aproximadamente $130{ }^{\circ} \mathrm{C}$ e verter a amostra na cápsula do conjunto até a marca de referência.

- Deixar esfriar o conjunto em temperatura ambiente por no mínimo $60 \mathrm{~min}$.

- Após $60 \mathrm{~min}$, adicionar água a $25 \pm 0,1^{\circ} \mathrm{C}$ no recipiente de banho-maria até cobrir a amostra e deixar em banho-maria, mantendo a temperatura da água a $25 \pm 0,1^{\circ} \mathrm{C}$ por no mínimo $90 \mathrm{~min}$.

- Após esse intervalo, acoplar o pino de giro no cilindro e efetuar a torção do asfalto no sentido de $180^{\circ}$ a $0^{\circ}$, durante um tempo entre $3 \mathrm{~s}$ e $5 \mathrm{~s}$.

- Em seguida, retirar imediatamente o pino de giro do cilindro, deixando o cilindro livre para que se inicie o retorno elástico do asfalto.

- Passados $30 \mathrm{~min} \pm 15 \mathrm{~s}$, fazer a leitura do ângulo recuperado "L" (em graus) e aplicar na fórmula para obter o retorno elástico "RE" (em \%).

$$
R E=\frac{(L \times 100)}{180}
$$

No modelo do aparelho utilizado nesta pesquisa, a recuperação elástica (em porcentagem) pode ser obtida diretamente na semi-coroa graduada, como pode ser visto na Figura 1.

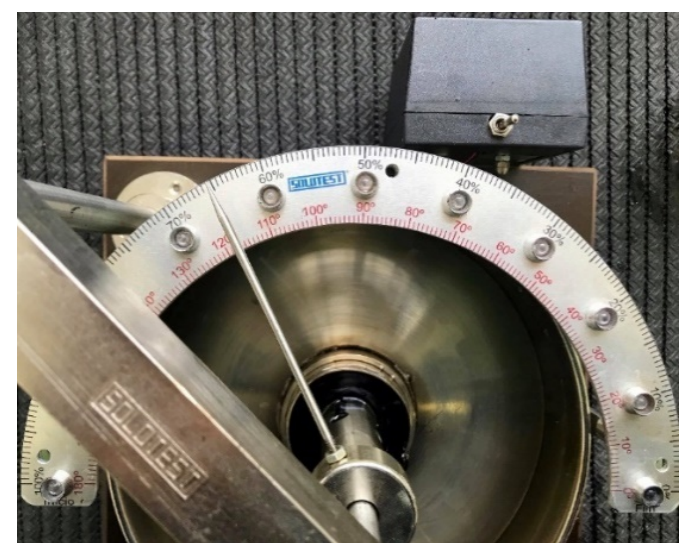

Figura 1. Semi-coroa graduada do torciômetro com a agulha indicando a recuperação elástica de uma amostra de asfalto modificado por polímero SBS

0 tempo de giro estipulado por norma é de 3 s a 5 s. É um giro rápido e que exige treino para fazê-lo dentro deste intervalo. Foram realizados ensaios com tempo de giro fora deste intervalo de tempo e observou-se se os resultados seriam afetados. Outra variável analisada foi em relação à temperatura do banho-maria em que a amostra permanece por no mínimo 90 minutos antes do giro. A norma cita que a amostra deve ser mantida em banho termostático de circulação a $25 \pm 0,1^{\circ} \mathrm{C}$ por $90 \mathrm{~min}$, para condicionamento da amostra, e, após o giro, apesar de não fazer nova referência ao controle temperatura, não orienta a suspensão da circulação de água. Portanto, realizou-se ensaios de retorno elástico com e sem controle de temperatura do banhomaria, após o giro da amostra, para verificar se a variação da temperatura de banho pode alterar os resultados do retorno elástico do asfalto. A norma não estabelece uma variação aceitável para resultados de retorno elástico encontrados. Sendo assim, análises estatísticas foram realizadas para a determinação da repetibilidade e reprodutibilidade dos resultados. 


\section{EXPERIMENTOS LABORATORIAIS}

\subsection{Caracterização das amostras}

Foram empregados 4 tipos de ligantes comercialmente conhecidos como: Poliflex 60/85 SBS, Poliflex 60/85 RET, TYREFLEX AB8 (com borracha de pneu moída) e o convencional, CAP $50 / 70$.

\subsection{Ensaio de recuperação elástica por torção}

Para análise da recuperação elástica foi utilizado o ensaio normalizado pela NLT 329/91. 0 medidor de recuperação elástica empregado é de fabricação da nacional (Figura 2).

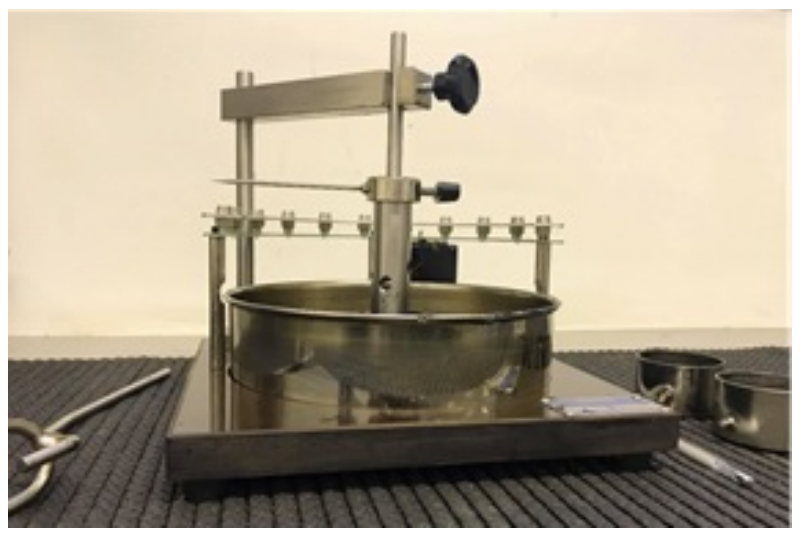

Figura 2. Torciômetro

\subsection{Planejamento do ensaio e métodos}

Os ensaios foram executados por dois operadores diferentes:

- Operador 01 foi responsável pela execução de 40 ensaios, com réplicas para cada tipo de asfalto (Poliflex 60/85 SBS, Poliflex 60/85 RET, TYREFLEX AB8 e CAP 50/70) de acordo com o procedimento descrito na norma, ou seja, com o tempo de giro entre $3 \mathrm{~s}$ e $5 \mathrm{~s}$, sem controlar a temperatura de banho da água durante o retorno elástico de $30 \mathrm{~min} \pm 15 \mathrm{~s} \mathrm{e}$ réplicas com controle de temperatura do banho durante o tempo de retorno elástico, em $25^{\circ} \mathrm{C} \pm 0,1^{\circ} \mathrm{C}$. Maiores detalhes podem ser visualizados na Figura 3.

- Operador 02 executou os ensaios num total de 60 vezes. Foram ensaiadas réplicas para cada tipo de asfalto modificado (Poliflex 60/85 SBS, Poliflex 60/85 RET e TYREFLEX AB8) com o tempo de giro entre $3 \mathrm{~s}$ e $5 \mathrm{~s}$, sem controlar a temperatura de banho durante o retorno elástico de $30 \mathrm{~min} \pm 15$ s e réplicas mantendo a temperatura de banho da água em $25 \pm 0,1^{\circ} \mathrm{C}$, durante todo o período de retorno elástico. Além disso, foram ensaiadas réplicas com asfalto modificado com tempo de giro entre $30 \mathrm{~s}$ a $50 \mathrm{~s}$, arbitrado para a pesquisa por ser de grandeza significantemente maior, sem o controle da temperatura durante o intervalo de retorno de $30 \mathrm{~min} \pm 15 \mathrm{~s}$ e com controle da temperatura $(25 \pm$ $0,1^{\circ} \mathrm{C}$ ). Maiores detalhes podem ser visualizados na Figura 4.

Para as inferências estatísticas, foi utilizado o teste t-Student, pois se trata de amostras pequenas ( $\mathrm{n} \leq 30)$, aleatórias e com observada distribuição normal (SPIEGEL, 1978). Para a análise comparativa entre as médias de retorno elástico obtidos para cada amostra de asfalto foi utilizado ANOVA. Para identificar quais amostras diferiram entre si foi utilizado o Teste de Tukey 
(TSD - Test Significant Difference). Para análise da Repetibilidade e Reprodutibilidade do ensaio, foi empregado o Método da análise de variância (ANOVA), que é uma das técnicas de Análise de Sistema de Medição (MSA) descritas no Manual desenvolvido pela Automotive Industry Action Group (AIAG). 0 método ANOVA é capaz de detectar eventuais interações entre as amostras e os operadores (AIAG, 2010).

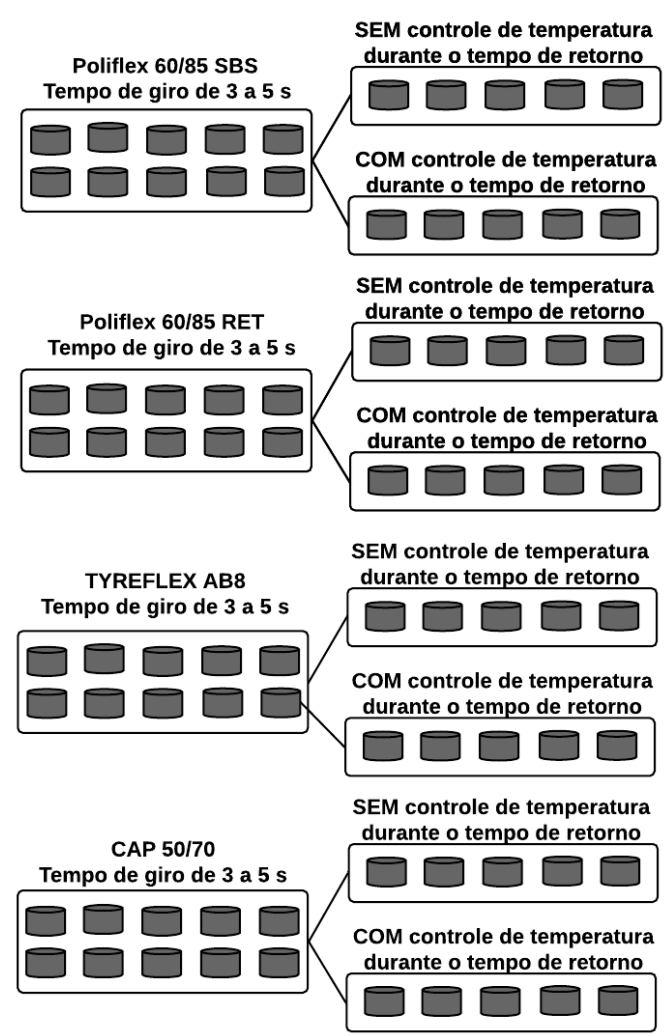

Figura 3. Organograma dos ensaios realizados pelo Operador 1

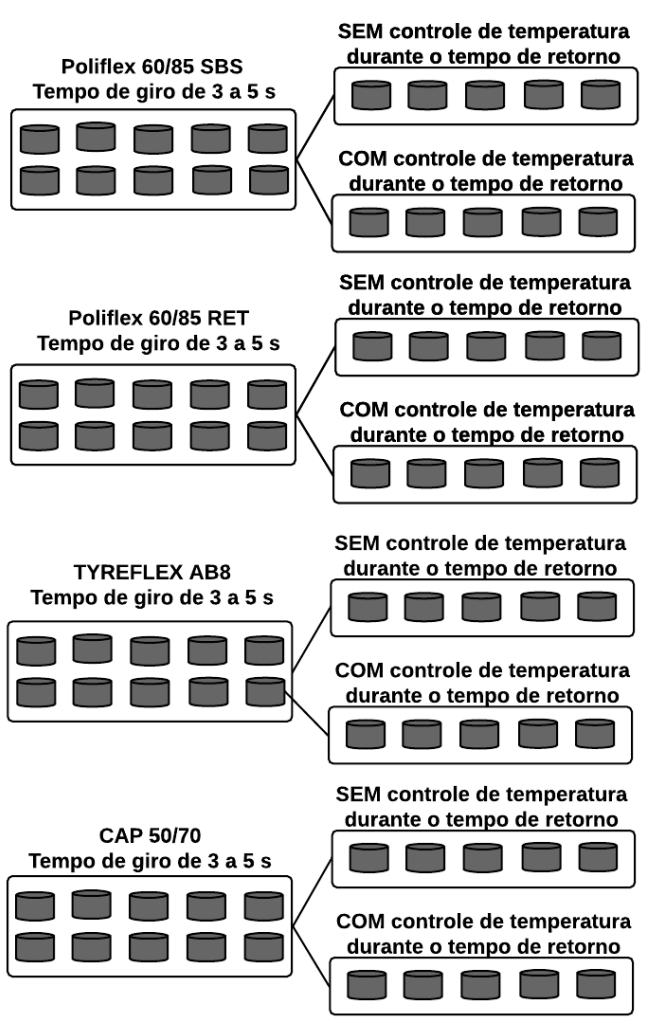

Figura 4. Organograma dos ensaios realizados pelo Operador 2

A Repetibilidade e Reprodutibilidade (R\&R) são medidas de precisão (incerteza) utilizadas para calcular a variabilidade de um Sistema de Medição. Um processo de medição é dito "confiável" se as medições puderem ser reproduzidas por diferentes pessoas, com diferentes equipamentos e em diferentes locais e situações (WONNACOTT \& WONNACOTT, 1990; PEDOTT, 2013). Dois aspectos importantes para assegurar o controle estatístico da qualidade em um processo de medição são a variabilidade natural das amostras e a outra associada ao erro de medição. 0 erro de medição pode ser dividido em duas partes, uma devido à variabilidade da Repetibilidade e outra devido à variabilidade da Reprodutibilidade (WESTERN ELETRIC, 1956; MONTGOMERY, 2004).

$$
\begin{gathered}
\sigma_{\text {Total }}{ }^{2}=\sigma_{\text {Amostra }}{ }^{2}+\sigma_{\text {Erro de medição }}{ }^{2} \\
\sigma_{\text {Erro de medição }}{ }^{2}=\sigma_{\text {Repetibilidade }}{ }^{2}+\sigma_{\text {Reprodutibilidade }}{ }^{2}
\end{gathered}
$$

onde: $\quad \sigma_{\text {Total }}{ }^{2}$ :

$\sigma_{\text {Amostras }}^{2}$ :

variância total observada;

$\sigma_{\text {Erro de medição }}^{2}$ :

variância natural entre as amostras;

$\sigma_{\text {Repetibilidade }}{ }^{2}$ : variância associada aos erros de medição; variância das medições obtidas por um mesmo operador com o mesmo equipamento de medição; 
$\boldsymbol{\sigma}_{\text {Reprodutibilidade }}{ }^{2}$ : variância das medições obtidas por operadores diferentes com o mesmo equipamento de medição.

No caso do método da ANOVA, a variância de Reprodutibilidade é dada por:

Onde: $\quad \sigma_{\beta}{ }^{2}$ : variância do operador;

$$
\sigma_{\text {Reprodutibilidade }}^{2}=\sigma_{\beta}^{2}+\sigma_{\tau \beta}^{2}
$$

$\sigma_{\tau \beta}{ }^{2}$ :variância da interação da amostra versus operador.

Logo, é possível determinar o desvio padrão associado aos efeitos de Repetibilidade e Reprodutibilidade juntos, chamado de $\left(\boldsymbol{\sigma}_{\text {Gage } R \boldsymbol{R}}\right)$ através de:

$$
\sigma_{\text {Gage } R R}=\sqrt{\sigma_{\text {Repetibilidade }}+\sigma_{\beta}+\sigma_{\tau \beta}}
$$

Em termos de desvio padrão, a soma das porcentagens de contribuição do sistema de medição (Gage RR) é dada por:

$$
\% \sigma_{R R}=\frac{\sigma_{\text {Gage RR }}}{\sigma_{\text {Total }}} * 100
$$

A soma das porcentagens de contribuição do sistema de medição (Gage RR) e das amostras não resulta $100 \%$, como ocorre para a variância. Isso decorre da formulação proposta no modelo ANOVA, com o desvio padrão total expresso como a raiz quadrada da soma das componentes parciais de variabilidade (PEDOTT \& FOGLIATTO, 2013).

O número de categorias distintas (NDC) permite identificar a habilidade de um sistema de medição em detectar diferenças entre as amostras de uma característica medida (AL-REFAIE \& BATA, 2010; GARCÍA \& RIO, 2013), ou seja, quantifica indiretamente a resolução do sistema de medição. 0 cálculo é feito através de:

$$
N D C=2 * \frac{\sigma_{\text {Amostra }}}{\sigma_{\text {Gage } R R}}
$$

Os critérios de aceitação baseados em porcentagem de $\sigma$ Gage R\&R, $\sigma 2$ Gage R\&R e NDC, definidos em AIAG (2010) são apresentados nas Tabelas 1, 2 e 3, respectivamente. Os resultados apresentados nesta pesquisa foram gerados através do software Minitab Statistical.

Tabela 1 - Componentes de variação $\left(\sigma^{2}\right)$

\begin{tabular}{ll}
\hline$\sigma$ Gage R\&R (\%) & Interpretação \\
\hline$<1 \%$ & Aceitável \\
$1 \%$ até $9 \%$ & Pode ser aceito, mas recomendam-se melhorias \\
$>9 \%$ & Inaceitável \\
\hline Fonte: Adaptado do Manual de MSA (2010)
\end{tabular}

Tabela 2 - Componentes de variação $(\sigma)$

\begin{tabular}{ll}
\hline$\sigma^{2}$ Gage R\&R (\%) & Interpretação \\
\hline$<10 \%$ & Aceitável \\
$10 \%$ até $30 \%$ & Pode ser aceito, mas recomendam-se melhorias \\
$>30 \%$ & Inaceitável \\
\hline Fonte: Adaptado do Manual de MSA (2010)
\end{tabular}

Tabela 3 - Interpretação do Número de Categorias Distintas

\begin{tabular}{ll}
\hline NDC & Interpretação \\
\hline$<2$ & O sistema não consegue diferenciar as amostras \\
2 até 5 & Pode ser usado, mas geralmente produz estimativas grosseiras \\
$>5$ & O sistema é aceitável e consegue diferenciar as amostras. \\
\hline Fonte: Adaptado do Manual de MSA (2010)
\end{tabular}




\section{RESULTADOS}

\subsection{Influência da temperatura do banho durante o retorno elástico}

Na Tabela 4, são apresentados os resultados do teste t-Student para as amostras de Poliflex 60/85 SBS, Poliflex 60/85 RET, TYREFLEX AB8 e CAP 50/70, ensaiadas pelo Operador 1 e na Tabela 5, as amostras de Poliflex 60/85 SBS, Poliflex 60/85 RET, TYREFLEX AB8, ensaiadas pelo Operador 2. Amostra I se refere ao conjunto de réplicas sem controle de temperatura durante o tempo de retorno. A amostra II se refere ao conjunto de réplicas com controle de temperatura durante o tempo de retorno elástico. Média se refere à média aritmética da recuperação elástica obtida no ensaio (em \%) para as réplicas sem e com controle de temperatura durante o tempo de retorno elástico (30 $\mathrm{min})$.

Tabela 4 - Resultados de retorno elástico (RE) sem e com controle de temperatura durante o tempo de retorno (tempo de giro de 3 s a 5 s) (Operador 1 )

\begin{tabular}{|c|c|c|c|c|c|c|c|}
\hline \multirow[t]{2}{*}{ Ligante } & \multicolumn{2}{|c|}{$\begin{array}{c}\text { Tamanho da } \\
\text { Amostra }\end{array}$} & \multicolumn{2}{|c|}{ Médias RE (\%) } & \multicolumn{2}{|c|}{$\begin{array}{c}\text { Desvio Padrão da } \\
\text { Amostra }\end{array}$} & \multirow[t]{2}{*}{ P-valor } \\
\hline & I & II & $I$ & II & $I$ & II & \\
\hline Poliflex 60/85 SBS & 5 & 5 & 76,3 & 74,4 & 1,9 & 1,1 & 0,0940 \\
\hline Poliflex 60/85 RET & 5 & 5 & 55,0 & 56,2 & 2,3 & 1,8 & 0,3896 \\
\hline TYREFLEX AB8 & 5 & 5 & 55,2 & 55,3 & 2,4 & 1,4 & 0,9376 \\
\hline CAP $50 / 70$ & 5 & 5 & 4,7 & 5,0 & 0,7 & 0,9 & 0,5777 \\
\hline
\end{tabular}

Observando o p-valor nos resultados do teste t-Student para tempo de giro entre $3 \mathrm{~s}$ a $5 \mathrm{~s}$ (Tabela 4), verifica-se que em todas as amostras ensaiadas pelo Operador 1 (Poliflex 60/85 SBS, Poliflex 60/85 RET, TYREFLEX AB8 e CAP 50/70), o nível descritivo é maior que 0,05, levando à aceitação da hipótese nula, ou seja, as médias dos tratamentos sem e com controle de temperatura são iguais. Este fato também foi observado em todas as amostras de ligantes modificados ensaiadas pelo Operador 2 (Tabela 5).

Tabela 5 - Resultados de retorno elástico (RE) sem e com controle de temperatura durante o tempo de retorno elástico (tempo de giro de $3 \mathrm{~s}$ a 5 s) (Operador 2)

\begin{tabular}{lccccccc}
\hline \multirow{2}{*}{ Ligante } & \multicolumn{2}{c}{$\begin{array}{c}\text { Tamanho da } \\
\text { Amostra }\end{array}$} & \multicolumn{2}{c}{ Médias RE (\%) } & \multicolumn{2}{c}{$\begin{array}{c}\text { Desvio Padrão da } \\
\text { Amostra }\end{array}$} & \multirow{2}{*}{ P-valor } \\
\cline { 2 - 6 } & I & II & I & II & I & II & \\
\hline Poliflex 60/85 SBS & 5 & 5 & 75,1 & 74,8 & 1,4 & 2,4 & 0,8184 \\
Poliflex 60/85 RET & 5 & 5 & 52,4 & 54,1 & 1,9 & 3,2 & 0,3421 \\
TYREFLEX AB8 & 5 & 5 & 52,9 & 52,5 & 1,0 & 1,4 & 0,6170 \\
\hline
\end{tabular}

Observa-se na Tabela 6 que a hipótese nula também é verdadeira para as amostras ensaiadas pelo Operador 2 com tempo de giro maior que o recomendado pela norma), ou seja, as médias de retorno elástico com e sem controle de temperatura do banho são estaticamente iguais. Logo, os resultados demonstram que controlar ou não a temperatura da água de banho duran-te o retorno elástico da amostra não influencia os resultados.

Vale ressaltar que nos meses em que foram ensaiadas as amostras, fevereiro a maio de 2018, a temperatura média na cidade e no laboratório esteve entre $21^{\circ} \mathrm{C}$ e $15^{\circ} \mathrm{C}$. Não se sabe se uma variação térmica ambiente maior alteraria os resultados. A perda de temperatura do banho, medida após os 30 minutos de recuperação, variou de $0,1^{\circ} \mathrm{C}$ a $0,5^{\circ} \mathrm{C}$.

Apesar de não terem sido observadas variações significativas nos resultados sem controle de temperatura, deve-se considerar que no campo e no dia a dia de uma usina, por exemplo, a 
temperatura ambiente pode variar intensamente e interferir nos resultados do ensaio. Portanto, é fortemente recomendável que a temperatura de banho seja mantida em $25^{\circ} \mathrm{C}$, durante os 30 minutos de retorno elástico, pois essa prática não requer nenhuma dificuldade e leva a uma maior confiabilidade dos resultados por manter a variável temperatura de banho constante.

Tabela 6 - Resultados de retorno elástico $(\mathrm{RE})$ sem e com controle de temperatura durante o tempo de retorno (tempo de giro de $30 \mathrm{~s}$ a $\mathbf{5 0}$ s) (Operador 2)

\begin{tabular}{lccccccc}
\hline \multirow{2}{*}{ Ligante } & \multicolumn{2}{c}{$\begin{array}{c}\text { Tamanho da } \\
\text { Amostra }\end{array}$} & \multicolumn{2}{c}{ Média da amostra } & $\begin{array}{c}\text { Desvio Padrão da } \\
\text { Amostra }\end{array}$ & \multirow{2}{*}{ P-valor } \\
\cline { 2 - 6 } & I & II & I & II & I & II & \\
\hline Poliflex 60/85 SBS & 5 & 5 & 70,6 & 73,2 & 4,7 & 2,8 & 0,3223 \\
Poliflex 60/85 RET & 5 & 5 & 52,1 & 56,0 & 3,3 & 2,4 & 0,0669 \\
TYREFLEX AB8 & 5 & 5 & 38,3 & 39,8 & 3,6 & 4,3 & 0,5695 \\
\hline
\end{tabular}

\subsection{Influência do tempo de giro}

Procedeu-se à inferência estatística nas réplicas em que a torção foi feita com menor velocidade, resultando num tempo de giro maior. Os resultados do teste de hipóteses podem ser vistos na Tabela 7, para as amostras de Poliflex 60/85 SBS, Poliflex 60/85 RET e TYREFLEX AB8, ensaiadas pelo Operador 2. Amostra I se refere ao conjunto de réplicas com tempo de giro de $3 \mathrm{~s} \mathrm{a} 5 \mathrm{~s}$, conforme orientação da NLT 329/91. A amostra II se refere ao conjunto de réplicas com tempo de giro de $30 \mathrm{~s}$ a $50 \mathrm{~s}$, arbitrado para a pesquisa. Média se refere à média aritmética da recuperação elástica obtida no ensaio (em \%) para as réplicas com tempo de giro de $3 \mathrm{~s}$ a 5 s e $30 \mathrm{~s}$ a $50 \mathrm{~s}$, respectivamente.

Tabela 7 - Comparação entre réplicas com tempo de giro de 3 a 5 s (I) e 30 a 50 s (II)

\begin{tabular}{l|c|c|c|c|c|c|c}
\hline \multirow{2}{*}{ Ligante } & \multicolumn{2}{|c|}{$\begin{array}{c}\text { Tamanho da } \\
\text { Amostra }\end{array}$} & \multicolumn{2}{c|}{ Média RE (\%) } & \multicolumn{2}{c}{$\begin{array}{c}\text { Desvio Padrão da } \\
\text { Amostra }\end{array}$} & \multirow{2}{*}{ P-valor } \\
\cline { 2 - 7 } & I & II & I & II & I & II & \\
\hline Poliflex 60/85 SBS & 10 & 10 & 75,0 & 71,9 & 1,9 & 3,9 & 0,0396 \\
\cline { 2 - 7 } $\begin{array}{l}\text { Poliflex 60/85 RET } \\
\text { TYREFLEX AB8 }\end{array}$ & 10 & 10 & 53,3 & 54,1 & 6,9 & 11,7 & 0,5657 \\
\cline { 2 - 7 } & 10 & 10 & 52,7 & 39,1 & 1,3 & 14,8 & $2,9 \mathrm{E}-09$ \\
\hline
\end{tabular}

Observando a Tabela 7, para o Poliflex 60/85 SBS e o Tyreflex AB8, respectivamente, o pvalor foi menor que o limite de 5\% (considerando índice de confiança de 95\%), indicando que a hipótese nula é falsa, logo, há diferença entre médias de ensaios feitos com os tempos de giros diferentes. Já para o Poliflex 60/85 RET a hipótese nula não pode ser rejeitada, considerando o p-valor de 0,5657 . Tal diferença pode indicar que os ligantes se comportaram de maneira diferente a tempos de torção diferentes. Portanto, o tempo de giro influencia os resultados de retorno elástico por torção. Considerando as variáveis estudadas, controle de temperatura do retorno elástico e tempo de giro, pode-se verificar que os padrões normativos do ensaio estão condizentes com o que foi verificado em laboratório.

\subsection{Considerações gerais sobre o ensaio}

Os resultados de retorno elástico obtidos pelo Operador 1 e analisados pelo teste ANOVA indicam haver diferenças entre as amostras de diferentes tipos de asfalto, considerando a população amostral de 10 réplicas para cada amostra, uma vez que o controle da temperatura não acarretou variações nas médias dos ligantes. 0 Teste de Tukey revelou que há diferença entre médias 
das amostras Poliflex 60/85 SBS, Poliflex 60/85 RET, TYREFLEX AB 8 e CAP 50/70. Portanto, os dois testes indicam que há diferenças de retorno elástico para cada tipo de ligante estudado.

O CAP 50/70 teve retorno elástico muito menor que os asfaltos modificados, cerca de 5\%, evidenciando a influência de modificadores no comportamento elástico dos ligantes. Observouse que durante os ensaios, após o giro, seu retorno se dava nos 5 minutos iniciais, permanecendo na mesma posição até a finalização do tempo exigido pela norma. Vale ressaltar que a NLT 329/91 foi desenvolvida para asfaltos modificados, o uso do ensaio para o ligante comum nesta pesquisa é de efeito comparativo.

0 asfalto Poliflex 60/85 SBS demonstrou maior retorno elástico para ambos os operadores, média de 75,4\% para o Operador 1 e 75,0 \% para o Operador 2 . Sobre o comportamento do asfalto nos ensaios, constatou-se que o retorno é imediato ao término do giro, apresentando maior velocidade nos primeiros segundos, cerca de $70 \%$ do retorno total.

O Poliflex 60/85 RET e TYREFLEX AB 8, apresentaram retorno elástico médios muito próximos, em torno de 55\%, tanto para o Operador 1 quanto para o Operador 2, embora o comportamento dos dois materiais no ensaio tenha sido distinto. Foi notado que no caso do asfalto modificado com polímero RET, a recuperação imediata ocorreu de forma mais lenta e distribuída ao longo do tempo de retorno normatizado.

0 asfalto-borracha apresentou média de retorno elástico das réplicas acima do mínimo de 50\%, especificado pelo DER/PR ES-P 28/05 para serviço de pavimentação. Para os demais tipos de asfalto pesquisados, ainda não há especificação de recuperação elástica por torção mínima em normas nacionais ou internacionais.

Observa-se também que nas amostras com tempo de giro entre $30 \mathrm{~s}$ a $50 \mathrm{~s}$ o desvio padrão foi elevado, ou seja, a amplitude do intervalo maior gerou maior dispersão nos resultados. 0 retorno elástico médio foi menor no caso do ligante modificado com SBS e principalmente no caso do asfalto-borracha, quando comparado ao retorno elástico das amostras ensaiadas com $3 \mathrm{~s}$ a $5 \mathrm{~s}$ de tempo de giro. Quanto ao Poliflex 60/85 RET as médias de retorno elástico com tempos de giro de $3 \mathrm{~s}$ a $5 \mathrm{~s}$ e de $30 \mathrm{~s}$ a $50 \mathrm{~s}$ podem ser consideradas estatisticamente iguais.

Outro ponto relevante observado nos experimentos é a inviabilidade de se obter uma amostra de asfalto modificado com viscosidade adequada ao vertimento na cápsula com temperaturas próximas a $130^{\circ} \mathrm{C}$, como especifica a norma. Para amostras de asfalto modificado com polímeros SBS e RET, foi necessária uma faixa de temperatura entre $155^{\circ} \mathrm{C}$ e $165^{\circ}$. Para o asfaltoborracha observaram-se temperaturas próximas a $175^{\circ} \mathrm{C}$.

\subsection{Repetibilidade e Reprodutibilidade do ensaio}

A Repetibilidade e Reprodutibilidade (R\&R) reflete a confiabilidade do método de medição. Na Tabela 8, são apresentados os principais resultados da aplicação do Método estatístico ANOVA para analisar a variabilidade do ensaio.

Observa-se que a maior fonte de variação do ensaio é a diferença entre as amostras, ou seja, decorrente do tipo de asfalto. Essa alta porcentagem de contribuição na variância (96,52\%) é muito boa, pois significa que o ensaio consegue distinguir amostras de asfaltos diferentes, com erro total em torno de 3,5\%.

A R\&R de medição deve ser o menor possível, pois demonstra que as variabilidades da Repetibilidade e Reprodutibilidade. A Repetibilidade e a Reprodutibilidade do ensaio representam juntas 3,48\% da variação total. De acordo com a Tabela 1, adaptada do Manual do MSA, em 
termos de variância $\left(\sigma^{2}\right)$ pela porcentagem de Gage $R \& R$ o ensaio pode ser aceito, pois apresenta valores entre 1\% e 9\%, porém são recomendadas melhorias.

Tabela 8 - Variação Total do ensaio

\begin{tabular}{lcccc}
\cline { 2 - 5 } & Variância $\left(\boldsymbol{\sigma}^{\mathbf{2}}\right)$ & \% de contribuição $\left(\boldsymbol{\sigma}^{\mathbf{2}}\right)$ & Desvio padrão $(\boldsymbol{\sigma})$ & \% de contribuição $(\boldsymbol{\sigma})$ \\
\hline Amostras & 146,3 & 96,52 & 12,1 & 98,24 \\
Repetibilidade & 3,9 & 2,56 & 2,0 & 15,99 \\
Reprodutibilidade & 1,4 & 0,93 & 1,2 & 9,64 \\
Total de R\&R (Gage R\&R) & 5,3 & 3,48 & 2,3 & 18,67 \\
\hline
\end{tabular}

A baixa variação de Reprodutibilidade (0,93\% do total) significa que o ensaio utilizado é capaz de reproduzir a mesma ordem de valores quando feito por diferentes pessoas. Ou seja, erros devido à Reprodutibilidade são inferiores a $1 \%$. A variação das medições realizadas por um mesmo operador, Repetibilidade, foi mais alta (2,55\% do total). Portanto, a maior fonte de erros relacionados ao ensaio é advinda das habilidades dos operadores na execução do ensaio. Logo, uma das formas de melhoria do Sistema de Medição é a aplicação de treinamento nos operadores.

Em termos de desvio padrão, analogamente à variância, o sistema pode ser considerado “aceitável”, pois o Gage R\&R está entre 10 e 30 \%. Como já mencionado, sugere-se melhorar a Repetibilidade, que foi a principal responsável pelo Gage R\&R de 18,67\%.

Na Tabela 9, é apresentada a variância de $R \& R$ para cada ligante estudado. Verifica-se que para asfalto Poliflex 60/85 SBS 100\% da variação dos valores são devido à Repetibilidade, ou seja, relativas à variação de valores quando o ensaio é executado por um mesmo operador. No caso do Poliflex 60/85 RET, a variância correspondente à Repetibilidade foi de 5,58\% que corresponde a aproximadamente $72 \%$ da variação total. Já para o asfalto TYREFLEX AB8, as fontes de erro foram mais equilibradas.

Tabela 9 - Componentes de variância para cada amostra de asfalto

\begin{tabular}{lcccccc}
\cline { 2 - 6 } & \multicolumn{2}{c}{ Poliflex 60/85 SBS } & \multicolumn{2}{c}{ Poliflex 60/85 RET } & \multicolumn{2}{c}{ TYREFLEX AB8 } \\
\cline { 2 - 7 } & Variância & \% de contribuição & Variância & \% de contribuição & Variância & \% de contribuição \\
\hline Repetibilidade & 3,38 & $100,00 \%$ & 5,58 & $71,71 \%$ & 2,37 & $44,06 \%$ \\
Reprodutibilidade & $-0,26 *$ & $0,00 \%$ & 2,20 & $28,29 \%$ & 3,01 & $55,94 \%$ \\
\hline
\end{tabular}

O Gráfico de interação Operador versus Amostra, apresentado na Figura 5, apresenta as médias das medições de cada Operador para os três tipos de asfaltos estudados. Quanto mais próxima as linhas dos Operadores 1 e 2, melhor é a Reprodutibilidade do ensaio, pois verifica-se que ambos obtêm valores da mesma ordem de grandeza. Há uma sobreposição de pontos no caso Poliflex 60/85 SBS, ou seja, não houve erro observado entre os valores obtidos de cada operador. No caso do Poliflex 60/85 RET e do TYREFLEX AB8, uma linha está acima da outra, indicando uma variação de Reprodutibilidade.

O Gráfico de Medições por Amostra (Figura 5) fornece uma análise geral para Repetibilidade e Reprodutibilidade do ensaio, pois apresenta a faixa de todas as medições para tipo de asfalto, considerando os dois operadores. Fica evidente as diferenças entre os valores de retorno elástico por torção dos diferentes tipos de asfalto. Além disso, observa-se que há maior dispersão nos resultados para o ligante modificado com polímero RET. 


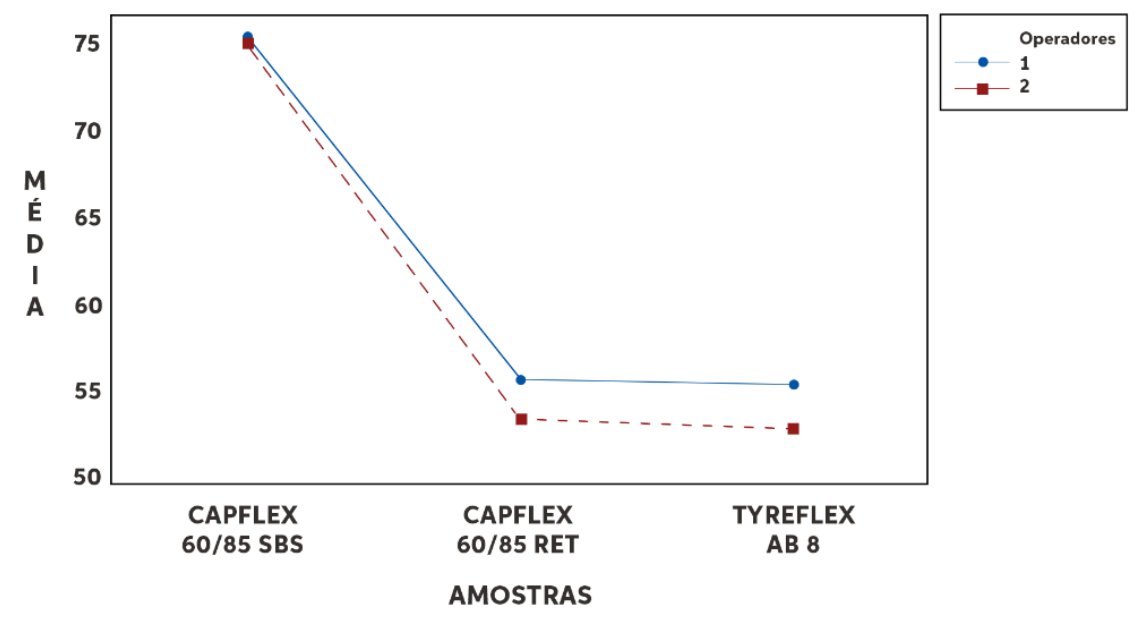

Figura 5. Gráfico de interação (Operador x Amostra)

O Gráfico de Medições por Amostra (Figura 6) fornece uma análise geral para Repetibilidade e Reprodutibilidade do ensaio, pois apresenta a faixa de todas as medições para tipo de asfalto, considerando os dois operadores. Fica evidente as diferenças entre os valores de retorno elástico por torção dos diferentes tipos de asfalto. Além disso, observa-se que há maior dispersão nos resultados para o ligante modificado com polímero RET.

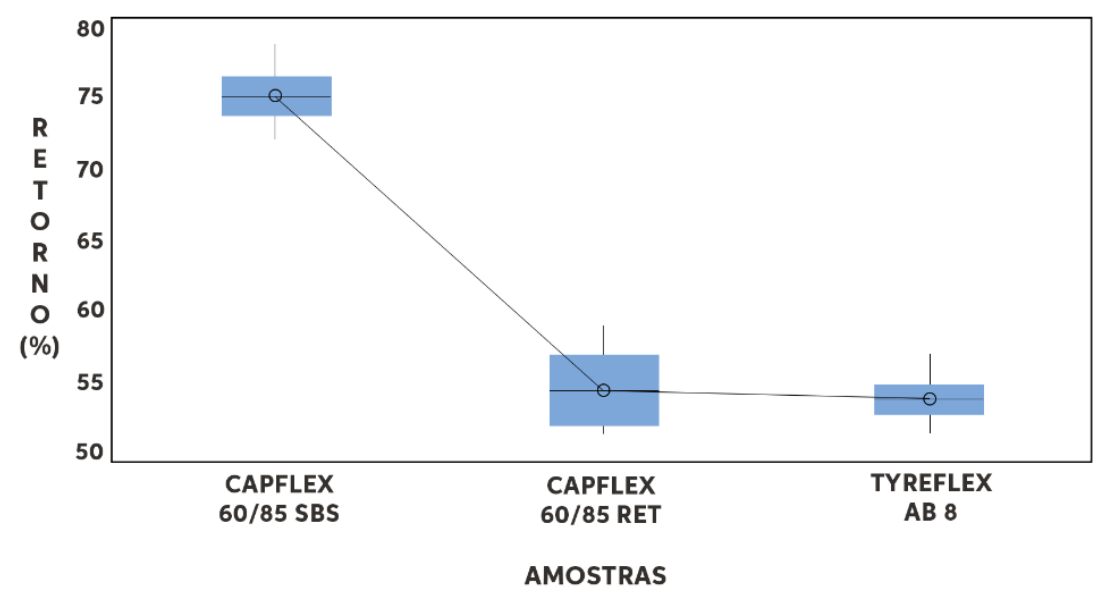

Figura 6. Gráfico de medições por amostra

0 boxplot da Figura 7 representa a amplitude de todas as medições e o ponto central representa a média geral de valores de retorno elástico obtidos para cada operador. Quanto mais paralela ao eixo for linha que liga esses pontos, mais próximas são medições dos operadores, ou seja, maior é a Reprodutibilidade do ensaio. Verifica-se que o intervalo das medições do segundo operador foi maior, resultando em certa inclinação da linha, ou seja, há maior dispersão nos resultados de seus ensaios.

Segundo o Manual do MSA, a variabilidade entre os resultados de ensaios realizados por diferentes operadores e/ou diferentes equipamentos, tende a ser maior que a variabilidade entre os resultados de ensaios realizados por um mesmo operador utilizando o mesmo equipamento. No entanto, neste estudo, notou-se maior erro de Repetibilidade provavelmente associado à maior amplitude dos resultados de retorno elástico do Operador 2 (Figura 6). 


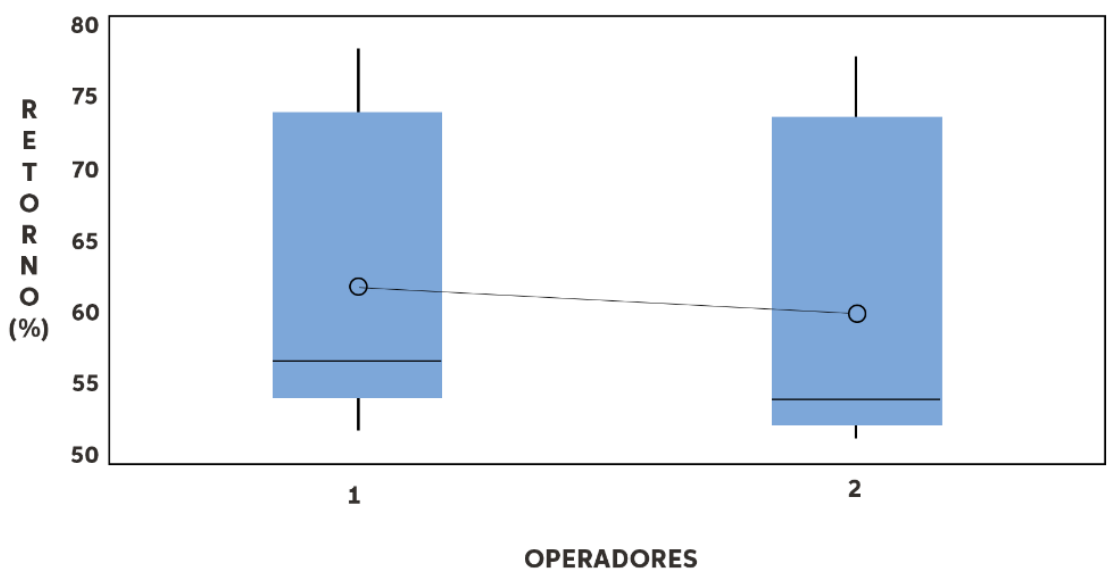

Figura 7. Gráfico de medições por operador

Além do parâmetro Gage R\&R foi calculado o Número de Categorias Distintas utilizado para aceitação de um Sistema de Medição. 0 ensaio estudado obteve valor de NDC igual a 7, ou seja, acima do limite de 5,0 estipulado para aceitação, segundo o Manual do MSA (Tabela 3). Isso indica que o sistema é capaz de diferenciar as diferentes amostras de asfalto avaliadas. Em outras palavras, indica que o ensaio é confiável para analisar o retorno elástico de diferentes tipos de asfaltos.

\section{CONCLUSÕES}

Com base nos resultados deste estudo, seguem as conclusões sobre o Ensaio de Retorno Elástico por Torção (NLT 329/91):

- Não houve influência da temperatura do banho durante o tempo de retorno elástico de 30 minutos, as médias com controle e sem controle de temperatura foram estatisticamente iguais para todas as amostras de asfalto estudadas, pois houve pequena variação da temperatura de banho. No entanto, deve-se considerar que a temperatura ambiente pode variar intensamente e interferir nos resultados do ensaio. Portanto, é fortemente recomendável que a temperatura de banho seja mantida em $25^{\circ} \mathrm{C}$, durante os 30 minutos de retorno elástico, pois essa prática não requer nenhuma dificuldade e leva a uma maior confiabilidade dos resultados;

- Houve influência do tempo de giro nas amostras estudadas, pois dois dos três asfaltos empregados na pesquisa apresentaram médias de retorno elástico menor quando o tempo giro foi maior do que o tempo padronizado pela NLT 329/91;

- ensaio pode ser considerado confiável, pois apresentou valores adequados para Repetibilidade e Reprodutibilidade, conforme os parâmetros encontrados no Manual do MSA;

- erro de Repetibilidade foi superior ao de Reprodutibilidade. Uma das formas de reduzir essa variação de resultados entre as réplicas do mesmo operador é fornecendo treinamento na execução do ensaio;

- asfalto que apresentou maior retorno elástico entre as amostras estudadas foi o modificado com polímero SBS. 0 asfalto modificado com polímero do tipo RET e o asfalto-borracha tiveram médias de retorno elástico próximas. 


\section{AGRADECIMENTOS}

Os autores agradecem à CBB Asfaltos pelo fornecimento do ligante asfáltico, à Fundação Araucária pelo suporte financeiro na forma de bolsas ao primeiro e ao segundo autor e à Universidade Estadual de Ponta Grossa pela infraestrutura para a realização da pesquisa.

\section{REFERÊNCIAS}

AUTOMOTIVE INDUSTRY ACTION GROUP (AIAG). Measurement systems analysis Reference Manual. 4ed. Chrysler, Ford, General Motors Supplier Quality Requirements Task Force, 2010.

Al-REFAIE, A. e N. BATA Evaluating measurement and process capabilities by GR\&R with four quality measures. Measurement: Journal of the International Measurement Confederation, v.43, p.842-851. 2010. https://doi.org/10.1016/j.measurement.2010.02.016

BATES, R. e R. WORCH Styrene-Butadiene Rubber Latex Modified Asphalt. Engineering Brief No. 39. Federal Aviation Administration, Washington, DC, 1987.

CENTRO DE ESTUDIOS DE CARRETERAS DEL CEDEX. Norma NLT-329/91, Recuperación Elástica por Torsión de Betunes Asfálticos Modificados. Espanha, 1991.

CONFEDERAÇÃO NACIONAL DO TRANSPORTE - CNT. Transporte rodoviário: por que os pavimentos das rodovias do Brasil não duram? - CNT, Brasília, 2017.

DEPARTAMENTO DE ESTRADAS DE RODAGEM DO PARANÁ - DER/PR. DER/PR ES-P 28/05. Pavimentação: Concreto Asfáltico Usinado a Quente com Asfalto Borracha. Curitiba, Paraná, 2005.

DEPARTAMENTO NACIONAL DE INFRAESTRUTURA DE TRANSPORTES - DNIT. NORMA DNIT 130/2010 - ME. Determinação da recuperação elástica de materiais asfálticos pelo ductilômetro - Método de Ensaio. Rio de Janeiro, 2004.

GARCIA, A. C. e A. G. RIO Number of distinct data categories and gage repeatability and reproducibility. A double (but single) requirement. Measurement: Journal of the International Measurement Confederation, v.46, p.2514-2518, 2013. https://doi.org/10.1016/j.measurement.2013.04.065

MONTGOMERY, D.C. Introdução ao controle estatístico da qualidade. 4⿳ạ. ed. Rio de Janeiro: LTC, 2004.

ONOFRE, F. C.; V. T. F. CASTELO BRANCO; J. B. SOARES e A. L. FAXINA Avaliação do Efeito de Ligantes Asfálticos Modificados na Resistência à Deformação Permanente de Misturas Asfálticas Densas. Transportes, v. 21, n. 3, p. 14-21, 2013. https://doi.org/10.4237/transportes.v21i3.685

PEDOTT, A. H. e F. S. FOGLIATTO Estudo de repetitividade e reprodutibilidade para dados funcionais. Produção, v.23, p.548560, 2013. http://dx.doi.org/10.1590/S0103-65132012005000087

SILVA, L.S.; M. M. C. FORTE; L. P. SPECHT e J. A. CERATTI Polímeros como modificadores asfálticos. Transportes, v. 10, n. 1, p. 84-106, 2002. https://doi.org/10.14295/transportes.v10i1.164

SPIEGEL, M. R. Probabilidade e Estatística. McGraw-Hill, São Paulo, 1978.

YILDIRIM, Y. Polymer Modified Asphalt Binders. Construction and Building Materials, v. 21, n. 1, p. 66-72, 2007. https://doi.org/10.1016/j.conbuildmat.2005.07.007

WESTERN ELECTRIC COMPANY. Statistical Quality Control Handbook. Easton, Pa.; Mack Printing Company, 1956.

WONNACOTT, T.H. e J. R. WONNACOTT Introductory Statistics. New York. John Wiley \& Sons, 1990. 